\title{
Certain Geometric Properties of Generalized Dini Functions
}

\author{
Muhey U. Din, ${ }^{1}$ Mohsan Raza $\left(\mathbb{D},{ }^{1}\right.$ Saqib Hussain $\mathbb{D D}^{2},{ }^{2}$ and Maslina Darus $\mathbb{D}^{3}$ \\ ${ }^{1}$ Department of Mathematics, Government College University, Faisalabad, Pakistan \\ ${ }^{2}$ Department of Mathematics, COMSATS University Islamabad, Abbottabad Campus, Pakistan \\ ${ }^{3}$ Faculty of Science and Technology, School of Mathematical Sciences, Universiti Kebangsaan Malaysia, \\ 43600 Bangi, Selangor, Malaysia
}

Correspondence should be addressed to Mohsan Raza; mohsan976@yahoo.com

Received 20 March 2018; Accepted 25 June 2018; Published 11 July 2018

Academic Editor: Mitsuru Sugimoto

Copyright ( $\odot 2018$ Muhey U. Din et al. This is an open access article distributed under the Creative Commons Attribution License, which permits unrestricted use, distribution, and reproduction in any medium, provided the original work is properly cited.

We are mainly interested in some geometric properties for the combinations of generalized Bessel functions of the first kind and their derivatives known as Dini functions. In particular, we study the starlikeness of order $\alpha$, convexity of order $\alpha$, and close-toconvexity of order $((1+\alpha) / 2)$ for normalized Dini function. We also study close-to-convexity with respect to certain star-like functions. Further, we obtain conditions on generalized Dini function to belong to the Hardy space $\mathscr{H}^{p}$.

\section{Introduction}

Let $\mathscr{A}$ be the class of functions $f$ of the form

$$
f(z)=z+\sum_{n=2}^{\infty} a_{n} z^{n}
$$

analytic in the open unit disc $\mathscr{U}=\{z:|z|<1\}$ and $\mathcal{S}$ denote the class of all functions in $\mathscr{A}$ which are univalent in $\mathscr{U}$. Let $\mathcal{S}^{*}(\alpha), \mathscr{C}(\alpha), \mathscr{K}(\alpha)$, and $\widetilde{\mathcal{S}^{*}}(\alpha)$ denote the classes of star-like, convex, close-to-convex and strongly star-like functions of order $\alpha$, respectively, and they are defined as

$$
\begin{aligned}
& \mathcal{S}^{*}(\alpha)=\left\{f: f \in \mathscr{A} \text { and } \operatorname{Re}\left(\frac{z f^{\prime}(z)}{f(z)}\right)>\alpha, z\right. \\
& \in \mathscr{U}, \alpha \in[0,1)\}, \\
& \mathscr{C}(\alpha)=\left\{f: f \in \mathscr{A} \text { and } \operatorname{Re}\left(1+\frac{z f^{\prime \prime}(\mathrm{z})}{f^{\prime}(z)}\right)>\alpha, z\right. \\
& \in \mathscr{U}, \alpha \in[0,1)\},
\end{aligned}
$$

$$
\begin{aligned}
& \mathscr{K}(\alpha)=\left\{f: f \in \mathscr{A} \text { and } \operatorname{Re}\left(\frac{z f^{\prime}(z)}{g(z)}\right)>\alpha, z\right. \\
& \left.\in \mathscr{U}, \alpha \in[0,1), g \in \mathcal{S}^{*}\right\}, \\
& \widetilde{\mathcal{S}^{*}}(\alpha)=\left\{f: f \in \mathscr{A} \text { and }\left|\arg \left(\frac{z f^{\prime}(z)}{f(z)}\right)\right|<\frac{\alpha \pi}{2}, z\right. \\
& \in \mathscr{U}, \alpha \in[0,1)\} .
\end{aligned}
$$

It is clear that

$$
\begin{aligned}
\widetilde{\mathcal{S}^{*}}(1) & =\mathcal{S}^{*}(0)=\mathcal{S}^{*}, \\
\mathscr{C}(0) & =\mathscr{C}, \\
\mathscr{K}(0) & =\mathscr{K} .
\end{aligned}
$$

If $f$ and $g$ are analytic functions, then the function $f$ is said to be subordinate to $g$, written as $f(z) \prec g(z)$, if there exist a Schwarz function $w$ which is analytic with $w(0)=0$ and $|w|<1$ such that $f(z)=g(w(z))$. Furthermore, if 
the function $g$ is univalent in $\mathcal{U}$ then we have the following equivalent relation:

$$
\begin{aligned}
& f(z) \prec g(z) \Longleftrightarrow \\
& f(0)=g(0), \\
& f(\mathcal{U}) \subset g(\mathcal{U}) .
\end{aligned}
$$

Special functions have great importance in pure and applied mathematics. The wide use of these functions has attracted many researchers to work on the different directions. Geometric properties of special functions such as hypergeometric functions, Bessel functions, Struve functions, Mittag-Leffler functions, Wright functions, and some other related functions are an ongoing part of research in geometric function theory. We refer to some geometric properties of these functions [1-10] and references therein.

Consider the second-order homogeneous differential equation

$$
z^{2} w^{\prime \prime}(z)+b z w^{\prime}(z)+\left(c z^{2}-v^{2}+(1-b) v\right) w(z)=0
$$

where $b, c$, and $v$ are complex numbers. The particular solution of the homogenous differential equation (6) is called the generalized Bessel functions of the first kind of order $v$. It is defined as

$$
w_{v, b, c}(z)=\sum_{n=0}^{\infty} \frac{(-c)^{n}(z / 2)^{2 n+v}}{n ! \Gamma(v+n+(b+1) / 2)},
$$

where $\Gamma$ (.) denotes the gamma function. The function $w_{v, b, c}$ unifies the Bessel, modified Bessel, and spherical Bessel functions.

\section{Special cases}

(1) For $b=c=1$, we have the Bessel functions of first kind of order $v$, defined as

$$
J_{v}(z)=\sum_{n=0}^{\infty} \frac{(-1)^{n}(z / 2)^{2 n+v}}{n ! \Gamma(v+n+1)} .
$$

(2) For $b=1, c=-1$, we obtain the modified Bessel functions of first kind of order $v$ whose series form is given as

$$
I_{v}(z)=\sum_{n=0}^{\infty} \frac{(z / 2)^{2 n+v}}{n ! \Gamma(v+n+1)} .
$$

(3) For $b=2, c=1$, we have the spherical Bessel functions of first kind of order $v$, given as

$$
j_{v}(z)=\sqrt{\frac{\pi}{2}} \sum_{n=0}^{\infty} \frac{(z / 2)^{2 n+v}}{n ! \Gamma(v+n+3 / 2)} .
$$

For more details about these functions, see $[5,11]$. Recently, Deniz et al. [12] studied the function $\varphi_{v, b, c}$ which is defined by the relation as

$\varphi_{v, b, c}(z)=2^{v} \Gamma\left(v+\frac{b+1}{2}\right) z^{1-v / 2} w_{v, b, c}\left(z^{1 / 2}\right)$ where $b, c, v \in \mathbb{C}$. Using the well-known Pochhammer symbol

$$
\begin{aligned}
(\gamma)_{n} & =\frac{\Gamma(\gamma+n)}{\Gamma(\gamma)} \\
& = \begin{cases}1, & n=0, \gamma \in \mathbb{C} \backslash\{0\}, \\
\gamma(\gamma+1) \ldots(\gamma+n-1), & n \in \mathbb{N}, \gamma \in \mathbb{C},\end{cases}
\end{aligned}
$$

we obtain the series form of the function $\varphi_{v, b, c}$ as

$$
\varphi_{v, b, c}(z)=z+\sum_{n=1}^{\infty} \frac{(-c)^{n}}{4^{n}(k)_{n} n !} z^{n+1}
$$

where $k=v+(b+1) / 2 \neq 0,-1,-2, \cdots$.

Bessel functions are indispensable in many branches of mathematics and applied mathematics. Thus, it is important to study their properties in many aspects. We consider the generalized Dini functions $d_{v, a, b, c}: \mathcal{U} \longrightarrow \mathbb{C}$ defined as

$$
\begin{aligned}
& d_{v, a, b, c}(z)=\frac{2^{v}}{a} \Gamma\left(v+\frac{b+1}{2}\right) \\
& \cdot z^{1-v / 2}\left((a-v) \varphi_{v, b, c}(\sqrt{z})+\sqrt{z} \varphi_{v, b, c}^{\prime}(\sqrt{z})\right)=z \\
& +\sum_{n=1}^{\infty} \frac{(-c)^{n}(2 n+a) \Gamma(v+1)}{a 4^{n} n ! \Gamma(v+n+1)} z^{n+1}=z \\
& +\sum_{n=1}^{\infty} \frac{(-c)^{n}(2 n+a)}{4^{n} n ! a(k)_{n}} z^{n+1},
\end{aligned}
$$

where $a \in \mathbb{R}^{+}, b \in \mathbb{R}, c \in \mathbb{C}$, and $v \in \mathbb{R}$. For $a=b=c=1$, we obtain the normalized Dini function of order $v$ of the form

$$
\begin{aligned}
d_{v}(z) & =z+\sum_{n=1}^{\infty} \frac{(-1)^{n}(2 n+1) \Gamma(v+1)}{4^{n} n ! \Gamma(v+n+1)} z^{n+1} \\
& =z+\sum_{n=1}^{\infty} \frac{(-1)^{n}(2 n+1) \Gamma(k)}{4^{n} n ! \Gamma(k+n)} z^{n+1} \\
& =z+\sum_{n=1}^{\infty} \frac{(-1)^{n}(2 n+1)}{4^{n} n !(k)_{n}} z^{n+1} .
\end{aligned}
$$

By putting $v=1 / 2$ and $v=3 / 2$, we obtain the following particular cases of normalized Dini functions

$$
\begin{aligned}
& d_{1 / 2}(z)=z \cos \sqrt{z} \\
& d_{3 / 2}(z)=3 \cos \sqrt{z}-\frac{3(z-2) \sin \sqrt{z}}{2 \sqrt{z}} .
\end{aligned}
$$

Recently Baricz et al. [13] studied the close-to-convexity of Dini functions and some monotonicity properties and functional inequalities for the modified Dini function are discussed in [5]. Further some geometric properties of Dini functions are studied in [14]. 
This paper studies the Dini function $d_{v, a, b, c}$ given by the power series (14). We determine the conditions on parameters that ensure the Dini function to be star-like of order $\alpha$, convex of order $\alpha$, and close-to-convex of order $((1+\alpha) / 2)$. We also study the convexity in the domain $\mathscr{U}_{1 / 2}=\{z$ : $|z|<1 / 2\}$. Sufficient conditions on univalency of an integral operator defined by Dini function are also studied. We find the conditions on normalized Dini function to belong to the Hardy space $\mathscr{H}^{p}$.

To prove our main results, we need the following lemmas.

Lemma 1 (see [15]). If $f \in \mathscr{A}$ satisfy $\left|f^{\prime}(z)-1\right|<1$ for each $z \in \mathscr{U}$, then $f$ is convex in $\mathscr{U}_{1 / 2}=\{z:|z|<1 / 2\}$.

Lemma 2 (see [16]). Let $\beta \in \mathbb{C}$ with $\operatorname{Re}(\beta)>0$, and $c \in \mathbb{C}$ with $|c| \leq 1, c \neq-1$. If $h \in \mathscr{A}$ satisfies

$$
\left.|c| z\right|^{2 \beta}+\left(1-|z|^{2 \beta} \frac{z h^{\prime \prime}(z)}{\beta h^{\prime}(z)}\right) \mid \leq 1, \quad z \in \mathcal{U}
$$

then the integral operator

$$
C_{\beta}(z)=\left\{\beta \int_{0}^{z} t^{\beta-1} h^{\prime}(t) d t\right\}^{1 / \beta}, \quad z \in \mathcal{U},
$$

is analytic and univalent in $\mathcal{U}$.

Lemma 3 (see [17]). Let $G(z)$ be convex and univalent in the open unit disc with condition $G(0)=1$. Let $F(z)$ be analytic in the open unit disc with condition $F(0)=1$ and $F \prec G$ in the open unit disc. Then, $\forall n \in \mathbb{N} \cup\{0\}$, we obtain

$$
\begin{aligned}
& (n+1) z^{-1-n} \int_{0}^{z} t^{n} F(t) d t \\
& \quad \prec(n+1) z^{-1-n} \int_{0}^{z} t^{n} G(t) d t .
\end{aligned}
$$

Lemma 4 (see [18]). If $f \in \mathscr{A}$ satisfies the inequality

$$
\left|z f^{\prime \prime}(z)\right|<\frac{1-\alpha}{4}, \quad(z \in \mathscr{U}, 0 \leq \alpha<1)
$$

then

$$
\operatorname{Re} f^{\prime}(z)>\frac{1+\alpha}{4}, \quad(z \in \mathcal{U}, 0 \leq \alpha<1) .
$$

Lemma 5 (see [19]). If the function $f(z)=z+a_{2} z^{2}+\ldots+a_{n} z^{n}+$ $\ldots$ is analytic in $\mathcal{U}$ and in addition $1 \geq 2 a_{2} \geq \ldots \geq n a_{n} \ldots \geq 0$ or $1 \leq 2 a_{2} \leq \ldots \leq n a_{n} \ldots \leq 2$, then $f$ is close-to-convex function with respect to the convex function $z \longrightarrow-\log (1-z)$. Moreover, if the odd function $g(z)=z+b_{3} z^{3}+\ldots+b_{2 n-1} z^{2 n-1}+$ $\ldots$ is analytic in $\mathcal{U}$ and if $1 \geq 3 b_{3} \geq \ldots \geq(2 n+1) b_{2 n+1} \ldots \geq 0$ or $1 \leq 3 b_{3} \leq \ldots \leq(2 n+1) b_{2 n+1} \ldots \leq 2$, then $g$ is univalent in U.

\section{Geometric Properties of Normalized Dini Functions}

Theorem 6. Let $a \in \mathbb{R}^{+}, b \in \mathbb{R}, c \in \mathbb{C}$, and $v \in \mathbb{R}$ with $\alpha \in[0,1)$ and $z \in \mathcal{U}$. Then the following assertions are true: (i) If $\left(2|c|+a|c|(4(k+1)-|c|)+4|c|^{2}+a|c|^{2}\right) /((4(k+$ 1) $\left.-|c|)(4 a k-2|c|-a|c|)-|c|^{2}(2+a)\right)<1-\alpha$, then $d_{v, a, b, c} \in \mathcal{S}^{*}(\alpha)$.

(ii) If $\left((4(k+1)-|c|)(4|c|+2 a|c|)+9|c|^{2}+2|c|^{2}(2+a)+\right.$ $\left.a|c|^{2}\right) /\left((4(k+1)-|c|)(4 a k-4|c|-2 a|c|)-4|c|^{2}-2|c|^{2}(2+\right.$ a) $\left.-a|c|^{2}\right)<1-\alpha$, then $d_{v, a, b, c} \in \mathscr{C}(\alpha)$.

(iii) If $\left((4(k+1)-|c|)(4|c|+2 a|c|)+9|c|^{2}+2|c|^{2}(2+a)+\right.$ $\left.a|c|^{2}\right) /\left(4 a k(4(k+1)-|c|)<(1-\alpha) / 4\right.$, then $d_{v, a, b, c} \in$ $\mathscr{K}((1+\alpha) / 2)$.

(iv) $\operatorname{If}(2|c|(k+1)+a|c|(k+1)) /(a k(4(k+1)-|c|))<1-\alpha$, then $d_{v, a, b, c} / z \in \mathscr{P}(\alpha)$.

Proof. We use the inequality $\left|z d_{v, a, b, c}^{\prime}(z) / d_{v, a, b, c}(z)-1\right|<1-\alpha$ to prove the starlikeness of order $\alpha$ for the function $d_{v, a, b, c}$. So by using the well-known triangle inequality

$$
\left|z_{1}+z_{2}\right| \leq\left|z_{1}\right|+\left|z_{2}\right|
$$

with the equality

$$
\begin{aligned}
\frac{\Gamma(v+1)}{\Gamma(v+n+1)} & =\frac{1}{(v+1)(v+2) \ldots(v+n)}=\frac{1}{(v+1)_{n}} \\
& =\frac{1}{(k)_{n}}, \quad n \in \mathbb{N},
\end{aligned}
$$

and the inequalities

$$
\begin{gathered}
2(n-1) !(k+1)_{n-1} \geq n(k+1)^{n-1}, \\
(n-1) !(k+1)_{n-1} \geq(k+1)^{n-1},
\end{gathered}
$$

$n \in \mathbb{N}$,

we obtain

$$
\begin{aligned}
\left|d_{v, a, b, c}^{\prime}(z)-\frac{d_{v, a, b, c}(z)}{z}\right| \leq \sum_{n=1}^{\infty} \frac{n(2 n+a)|c|^{n}}{a 4^{n} n !(k)_{n}} \\
\leq \frac{|c|}{2 a k}+\frac{|c|}{4 k}+\frac{|c|}{a k} \sum_{n=2}^{\infty}\left(\frac{|c|}{4(k+1)}\right)^{n-1} \\
\quad+\frac{|c|}{4 k} \sum_{n=2}^{\infty}\left(\frac{|c|}{4(k+1)}\right)^{n-1} \\
=\frac{2|c|+a|c|(4(k+1)-|c|)+|c|^{2}(4+a)}{4 a k(4(k+1)-|c|)} .
\end{aligned}
$$

Furthermore, if we use reverse triangle inequality

$$
\left|z_{1}+z_{2}\right| \geq|| z_{1}|-| z_{2}||
$$

and the inequalities

$$
\begin{aligned}
& n !(k+1)_{n-1} \geq n(k+1)^{n-1}, \\
& n !(k+1)_{n-1} \geq(k+1)^{n-1},
\end{aligned}
$$


then we get

$$
\begin{aligned}
& \left|\frac{d_{v, a, b, c}^{\prime}(z)}{z}\right|=\left|1+\sum_{n=1}^{\infty} \frac{(-c)^{n}(2 n+a)}{\left.a 4^{n} n !\left(k_{n}\right)\right)} z^{n}\right| \\
& \geq 1-\frac{|c|}{2 a k}+\frac{|c|}{4 k}+\frac{|c|}{2 a k} \sum_{n=2}^{\infty}\left(\frac{|c|}{4(k+1)}\right)^{n-1} \\
& \quad+\frac{|c|}{4 k} \sum_{n=2}^{\infty}\left(\frac{|c|}{4(k+1)}\right)^{n-1} \\
& =\frac{-|c|^{2}(2+a)+(4(k+1)-|c|)(4 a k-2|c|-a|c|)}{4 a k(4(k+1)-|c|)}
\end{aligned}
$$

By combining inequalities (25) and (28), we get

$$
\begin{aligned}
& \left|\frac{z d_{v, a, b, c}^{\prime}(z)}{d_{v, a, b, c}(z)}-1\right| \\
& \leq \frac{2|c|+a|c|(4(k+1)-|c|)+|c|^{2}(4+a)}{-|c|^{2}(2+a)+(4(k+1)-|c|)(4 a k-2|c|-a|c|)} \\
& <1-\alpha .
\end{aligned}
$$

So, $d_{v, a, b, c}$ is star-like function of order $\alpha$, where $0 \leq \alpha<1-$ $\left(2|c|+a|c|(4(k+1)-|c|)+|c|^{2}(4+a)\right) /((4(k+1)-|c|)(4 a k-$ $\left.2|c|-a|c|)-|c|^{2}(2+a)\right)$.

(ii) To prove the convexity of order $\alpha$ of function $d_{v, a, b, c}$, we have to show that $\left|z d_{v, a, b, c}^{\prime \prime}(z) / d_{v, a, b, c}^{\prime}(z)\right|<1-\alpha$. By using the well-known triangle inequality

$$
\left|z_{1}+z_{2}\right| \leq\left|z_{1}\right|+\left|z_{2}\right|
$$

with equality

$$
\begin{aligned}
\frac{\Gamma(v+1)}{\Gamma(v+n+1)} & =\frac{1}{(v+1)(v+2) \ldots(v+n)}=\frac{1}{(v+1)_{n}} \\
& =\frac{1}{(k)_{n}}, \quad n \in \mathbb{N},
\end{aligned}
$$

$$
\begin{aligned}
\left|d_{v, a, b, c}^{\prime}(z)\right| & \geq 1-\sum_{n=1}^{\infty} \frac{(2 n+a)(n+1)}{a 4^{n} n !}|c|^{n} \\
& \leq 1-\frac{|c|}{a k}-\frac{|c|}{2 k}-\frac{|c|^{2}}{a k(4(k+1)-|c|)}-\frac{(2+a)|c|^{2}}{2 a k(4(k+1)-|c|)}-\frac{|c|^{2}}{4 k(4(k+1)-|c|)} \\
& =\frac{(4(k+1)-|c|)(4 a k-4|c|-2 a|c|)-4|c|^{2}-2|c|^{2}(2+a)-a|c|^{2}}{4 a k(4(k+1)-|c|} .
\end{aligned}
$$

By combining inequalities (33) and (37), we have

$$
\left|\frac{z d_{v, a, b, c}^{\prime \prime}(z)}{d_{v, a, b, c}^{\prime}(z)}\right| \leq \frac{(4(k+1)-|c|)(4|c|+2 a|c|)+9|c|^{2}+2|c|^{2}(2+a)+a|c|^{2}}{(4(k+1)-|c|)(4 a k-4|c|-2 a|c|)-4|c|^{2}-2|c|^{2}(2+a)-a|c|^{2}}<1-\alpha
$$


This implies that $d_{v, a, b, c}$ is convex function of order $\alpha$, where $0 \leq \alpha<1-\left((4(k+1)-|c|)(4|c|+2 a|c|)+9|c|^{2}+2|c|^{2}(2+a)+\right.$ $\left.a|c|^{2}\right) /\left((4(k+1)-|c|)(4 a k-4|c|-2 a|c|)-4|c|^{2}-2|c|^{2}(2+\right.$ a) $\left.-a|c|^{2}\right)$.

(iii) Using inequality (33) and Lemma 4, we have

$$
\begin{aligned}
& \left|z d_{v, a, b, c}^{\prime \prime}(z)\right| \\
& \leq \frac{(4(k+1)-|c|)(4|c|+2 a|c|)+9|c|^{2}+2|c|^{2}(2+a)+a|c|^{2}}{4 a k(4(k+1)-|c|} \\
& <\frac{1-\alpha}{4},
\end{aligned}
$$

where $0 \leq \alpha<1-4\left(\left((4(k+1)-|c|)(4|c|+2 a|c|)+9|c|^{2}+2|c|^{2}(2+\right.\right.$ $\left.a)+a|c|^{2}\right) /(4 a k(4(k+1)-|c|))$. This shows that $d_{v, a, b, c} \in \mathscr{K}((1+$ $\alpha) / 2)$. Therefore $\operatorname{Re}\left(d_{v, a, b, c}^{\prime}(z)\right)>(1+\alpha) / 2$.

(iv) To prove that $d_{v, a, b, c} / z \in \mathscr{P}(\alpha)$, we have to show that $|g(z)-1|<1$, where $g(z)=\left(d_{v, a, b, c}(z) /(z-\alpha)\right) /(1-\alpha)$. By using the well-known triangle inequality

$$
\left|z_{1}+z_{2}\right| \leq\left|z_{1}\right|+\left|z_{2}\right|
$$

with the equality

$$
\begin{array}{r}
\frac{\Gamma(v+1)}{\Gamma(v+n+1)}=\frac{1}{(v+1)(v+2) \ldots(v+n)}=\frac{1}{(v+1)_{n}}, \\
n \in \mathbb{N},
\end{array}
$$

and the inequality

$$
(n-1) !(k+1)_{n-1} \geq(k+1)^{n-1}, \quad n \in \mathbb{N},
$$

we have

$$
\begin{aligned}
|g(z)-1|= & \left|\frac{1}{1-\alpha} \sum_{n=1}^{\infty} \frac{(-c)^{n}(2 n+a)}{a 4^{n} n !(k)_{n}} z^{n}\right| \\
\leq & \frac{1}{(1-\alpha)} \frac{2|c|}{4 a k} \sum_{n=1}^{\infty}\left(\frac{|c|}{4(k+1)}\right)^{n-1} \\
& +\frac{1}{(1-\alpha)} \frac{|c|}{4 k} \sum_{n=1}^{\infty}\left(\frac{|c|}{4(k+1)}\right)^{n-1} \\
= & \frac{1}{(1-\alpha)} \frac{2|c|(k+1)+a|c|(k+1)}{a k(4(k+1)-|c|)} .
\end{aligned}
$$

Therefore, $d_{v, a, b, c} / z \in \mathscr{P}(\alpha)$ for $0<\alpha<1-(2|c|(k+1)+$ $a|c|(k+1)) /(a k(4(k+1)-|c|))$.

Corollary 7. By using Theorem 6 assertions (i) and (iv), we get the following corollary:

(i) If $0 \leq \alpha<\alpha_{1}$ where $\alpha_{1} \simeq 0.333333333 \ldots$, then $d_{1 / 2} \in$ $\mathcal{S}^{*}(\alpha)$.

(ii) If $0 \leq \alpha<\alpha_{2}$ where $\alpha_{2} \simeq 0.7727272727 \ldots$, then $d_{3 / 2} \epsilon$ $\mathcal{S}^{*}(\alpha)$.

(ii) If $0 \leq \alpha<\alpha_{3}$ where $\alpha_{3} \simeq 0.44444444 \ldots$, then $d_{1 / 2} / z \in$ $\mathscr{P}(\alpha)$.

(iii) If $0 \leq \alpha<\alpha_{4}$ where $\alpha_{4} \simeq 0.67692307 \ldots$, then $d_{3 / 2} / z \in$ $\mathscr{P}(\alpha)$.
Putting $\alpha=0$ in Theorem 6, we have the following results.

Corollary 8. Let $a \in \mathbb{R}^{+}, b \in \mathbb{R}, c \in \mathbb{C}$, and $v \in \mathbb{R}$. Then the following assertions are true.

(i) If $k>(8|c|(a+1)-4 a(4-|c|)+(\{8|c|(a+1)-4 a(4-$ $\left.\left.|c|)\}^{2}+64 a\left\{2|c|(4-|c|)(a+1)+2|c|^{2}(a+3)+2|c|\right\}\right)^{1 / 2}\right) / 32 a$, then $d_{v, a, b, c} \in \mathcal{S}^{*}$.

(ii) If $k>(4(8|c|+4 a|c|)-4 a(4-|c|)+(\{4(8|c|+4 a|c|)-$ $\left.\left.4 a(4-|c|)\}^{2}+64 a\left\{(4-|c|)(8|c|+4 a|c|)+|c|^{2}(6 a+21)\right\}\right)^{1 / 2}\right) / 32 a$, then $d_{v, a, b, c} \in \mathscr{C}$.

(iii) If $k>(8|c|(a+2)-a(4-|c|)+(\{8|c|(a+2)-a(4-$ $\left.\left.|c|)\}^{2}+16 a\left\{2|c|(a+2)(4-|c|)+|c|^{2}(3 a+13)\right\}\right)^{1 / 2}\right) / 8 a$, then $d_{v, a, b, c} \in \mathscr{K}(1 / 2)$.

(iv) If $k>(|c|(a+2)-a(4-|c|)+$ $\left.\sqrt{\{c \mid(a+2)-a(4-|c|)\}^{2}+16 a|c|(a+2)}\right) / 8 a, \quad$ then $d_{v, a, b, c} / z \in \mathscr{P}$.

Corollary 9. By putting $a=b=c=1$ and $k=v+1$ in Corollary 8, we obtain the following results:

(i) If $v>0.453768227$, then $d_{v} \in \mathcal{S}^{*}$.

(ii) If $v>2.406035949$, then $d_{v} \in \mathscr{C}$.

(iii) If $v>5.548088707$, then $d_{v} \in \mathscr{K}(1 / 2)$.

(iv) If $v>-0.8660254038$, then $d_{v} / z \in \mathscr{P}$.

Remark 10. It is easy to see that our results improve the results of [14] as special cases of parameters. Some particular cases regarding Corollary 9 are given below.

Corollary 11. (i) If $v=1 / 2$, then $d_{1 / 2}(z)=z \cos \sqrt{z} \in \mathcal{S}^{*}$.

(ii) If $v=3 / 2$, then $d_{3 / 2}(z)=3 \cos \sqrt{z}-(3(z-$ 2) $\sin \sqrt{z}) / 2 \sqrt{z} \in \mathcal{S}^{*}$.

(iii) If $v=1 / 2$, then $\left(d_{1 / 2} / z\right)(z)=\cos \sqrt{z} \in \mathscr{P}$.

(iv) If $v=3 / 2$, then $d_{3 / 2}(z) / z=(6 \sqrt{z} \cos \sqrt{z}-3(z-$ 2) $\sin \sqrt{z}) / 2 z^{3 / 2} \in \mathscr{P}$.

Theorem 12. Let $a \in \mathbb{R}^{+}, b \in \mathbb{R}, c \in \mathbb{C}$, and $v \in \mathbb{R}$. If $((4(k+$ $\left.1)-|c|)(|c|(a+4))+|c|^{2}(a+6)+4 a|c|(k+1)\right) /(4 a k(4(k+1)-$ $|c|))<1$, then $d_{v, a, b, c}$ is convex in $\mathscr{U}_{1 / 2}$.

Proof. By using the well-known triangle inequality

$$
\left|z_{1}+z_{2}\right| \leq\left|z_{1}\right|+\left|z_{2}\right|
$$

with the equality $\Gamma(v+1) / \Gamma(v+n+1)=1 /((v+1)(v+2) \ldots(v+$ $n))=1 /(v+1)_{n}, n \in \mathbb{N}$, and the inequalities

$$
\begin{array}{cl}
2 n !(k+1)_{n-1} \geq n^{2}(k+1)^{n-1}, & n \in \mathbb{N}, \\
n !(k+1)_{n-1} \geq n(k+1)^{n-1}, & n \in \mathbb{N}, \\
n !(k+1)_{n-1} \geq(k+1)^{n-1}, & n \in \mathbb{N},
\end{array}
$$

we obtain

$$
\begin{aligned}
& \left|d_{v, a, b, c}^{\prime}(z)-1\right| \leq \sum_{n=1}^{\infty} \frac{2 n^{2}+n(a+2)+a}{a 4^{n} n !(k)_{n}}|c|^{n} \\
& \leq \frac{|c|}{2 a k}+\frac{|c|}{a k} \sum_{n=2}^{\infty}\left(\frac{|c|}{4(k+1)}\right)^{n-1}+\frac{(a+2)|c|}{4 a k}
\end{aligned}
$$




$$
\begin{gathered}
+\frac{(a+2)|c|}{4 a k} \sum_{n=2}^{\infty}\left(\frac{|c|}{4(k+1)}\right)^{n-1}+\frac{|c|}{4 k} \sum_{n=1}^{\infty}\left(\frac{|c|}{4(k+1)}\right)^{n-1} \\
=\frac{(4(k+1)-|c|)(|c|(a+4))+|c|^{2}(a+6)+4 a|c|(k+1)}{4 a k(4(k+1)-|c|)} .
\end{gathered}
$$

In view of Lemma $1, d_{v, a, b, c}$ is convex in $\mathscr{U}_{1 / 2}$, if $((4(k+1)-$ $\left.|c|)(|c|(a+4))+|c|^{2}(a+6)+4 a|c|(k+1)\right) /(4 a k(4(k+1)-|c|))<1$, but this is true under the hypothesis.

Remark 13. When we put $a=b=c=1$, our results are better than [14].

Consider the integral operator $\mathscr{F}_{\beta}: \mathscr{U} \longrightarrow \mathbb{C}$, where $\beta \in$ $\mathbb{C}, \beta \neq 0$,

$$
\mathscr{F}_{\beta}(z)=\left\{\beta \int_{0}^{z} t^{\beta-2} d_{v, a, b, c}(t)\right\}^{1 / \beta}, \quad z \in \mathcal{U} .
$$

Here $\mathscr{F}_{\beta} \in \mathscr{A}$. In the next theorem, we obtain the conditions so that $\mathscr{F}_{\beta}$ is univalent in $\mathcal{U}$.

Theorem 14. Let $a \in \mathbb{R}^{+}, b \in \mathbb{R}, c \in \mathbb{C}$, and $v \in \mathbb{R}$. Let $\left(2|c|+a|c|(4(k+1)-|c|)+4|c|^{2}+a|c|^{2}\right) /\left(-|c|^{2}(2+a)+(4(k+\right.$ $1)-|c|)(4 a k-2|c|-a|c|))<1$ and suppose that $M$ is a positive real number such that $\left|d_{v, a, b, c}(z)\right| \leq M$ in the open unit disc. If

$$
\begin{aligned}
& |\beta-1| \\
& +\frac{2|c|+a|c|(4(k+1)-|c|)+4|c|^{2}+a|c|^{2}}{(4(k+1)-|c|)(4 a k-2|c|-a|c|)-|c|^{2}(2+a)} \\
& +\frac{M}{|\beta|} \leq 1
\end{aligned}
$$

then $\mathscr{F}_{\beta}$ is univalent in $\mathcal{U}$.

Proof. A calculation gives us

$$
\frac{z \mathscr{F}_{\beta}^{\prime \prime}(z)}{\mathscr{F}_{\beta}^{\prime}(z)}=\frac{z d_{v, a, b, c}^{\prime}(z)}{d_{v, a, b, c}(z)}+\frac{z^{\beta-1}}{\beta} d_{v, a, b, c}(z)+\beta-2,
$$

$$
z \in \mathscr{U}
$$

Since $d_{v, a, b, c} \in \mathscr{A}$, then, by the Schwarz Lemma, triangle inequality, and (29), we obtain

$$
\begin{aligned}
& \left(1-|z|^{2}\right)\left|\frac{z \mathscr{F}_{\beta}^{\prime \prime}(z)}{\mathscr{F}_{\beta}^{\prime}(z)}\right| \leq\left(1-|z|^{2}\right)\{|\beta-1| \\
& \left.+\left|\frac{z d_{v, a, b, c}^{\prime}(z)}{d_{v, a, b, c}(z)}-1\right|+\frac{|z|^{\mathscr{R}(\beta)}}{|\beta|}\left|\frac{d_{v, a, b, c}(z)}{z}\right|\right\} \leq(1 \\
& \left.-|z|^{2}\right)\{|\beta-1| \\
& +\frac{2|c|+a|c|(4(k+1)-|c|)+4|c|^{2}+a|c|^{2}}{-|c|^{2}(2+a)+(4(k+1)-|c|)(4 a k-2|c|-a|c|)} \\
& \left.+\frac{M}{|\beta|}\right\} \leq 1 .
\end{aligned}
$$

This shows that the given integral operator satisfies Becker's criterion for univalence, and hence $\mathscr{F}_{\beta}$ is univalent in $\mathcal{U}$.

\section{Strong Starlikeness}

In this section, we are mainly interested about some sufficient conditions under which the normalized Dini function belongs to the class of strong starlikeness of order $\alpha$.

Theorem 15. Let $a \in \mathbb{R}^{+}, b \in \mathbb{R}, c \in \mathbb{C}$, and $v \in \mathbb{R}$. If $((4(k+$ $\left.1)-|c|)(|c|(a+4))+|c|^{2}(a+6)+4 a|c|(k+1)\right) /(4 a k(4(k+1)-$ $|c|))<1$, then $d_{v, a, b, c} \in \overline{\mathcal{S}^{*}}(\alpha)$, where

$$
\alpha=\frac{2}{\pi} \arcsin \left(\varkappa \sqrt{1-\frac{\varkappa^{2}}{4}}+\frac{\varkappa}{4} \sqrt{1-\varkappa^{2}}\right),
$$

and $\varkappa=\left((4(k+1)-|c|)(|c|(a+4))+|c|^{2}(a+6)+4 a|c|(k+\right.$ $1)) /(4 a k(4(k+1)-|c|))$.

Proof. By using the well-known triangle inequality

$$
\left|z_{1}+z_{2}\right| \leq\left|z_{1}\right|+\left|z_{2}\right|
$$

with the equality $\Gamma(v+1) / \Gamma(v+n+1)=1 /((v+1)(v+2) \ldots(v+$ $n))=1 /(v+1)_{n}, n \in \mathbb{N}$, and the inequalities

$$
\begin{array}{cc}
2 n !(k+1)_{n-1} \geq n^{2}(k+1)^{n-1}, & n \in \mathbb{N}, \\
n !(k+1)_{n-1} \geq n(k+1)^{n-1}, & n \in \mathbb{N}, \\
n !(k+1)_{n-1} \geq(k+1)^{n-1}, & n \in \mathbb{N},
\end{array}
$$

we obtain

$$
\begin{aligned}
& \left|d_{v, a, b, c}^{\prime}(z)-1\right| \leq \sum_{n=1}^{\infty} \frac{2 n^{2}+n(a+2)+a}{a 4^{n} n !(k)_{n}}|c|^{n} \\
& \leq \frac{|c|}{2 a k}+\frac{|c|}{a k} \sum_{n=2}^{\infty}\left(\frac{|c|}{4(k+1)}\right)^{n-1}+\frac{(a+2)|c|}{4 a k} \\
& \quad+\frac{(a+2)|c|}{4 a k} \sum_{n=2}^{\infty}\left(\frac{|c|}{4(k+1)}\right)^{n-1}+\frac{|c|}{4 k} \sum_{n=1}^{\infty}\left(\frac{|c|}{4(k+1)}\right)^{n-1} \\
& =\frac{(4(k+1)-|c|)(|c|(a+4))+|c|^{2}(a+6)+4 a|c|(k+1)}{4 a k(4(k+1)-|c|)} \\
& =\varkappa .
\end{aligned}
$$

For $0<\varkappa \leq 1$ and from (54), we concluded that

$$
\begin{aligned}
d_{v, a, b, c}^{\prime} & <1+\varkappa z \Longrightarrow \\
\left|\arg \left(d_{v, a, b, c}^{\prime}(z)\right)\right| & <\arcsin \varkappa .
\end{aligned}
$$

With the help of Lemma 3, take $n=0$ with $F(z)=d_{v, a, b, c}^{\prime}(z)$ and $G(z)=1+\varkappa z$, and we get

$$
\frac{d_{v, a, b, c}^{\prime}}{z} \prec 1+\frac{\varkappa}{2} z \text {. }
$$


As a result

$$
\left|\arg \left(\frac{d_{v, a, b, c}(z)}{z}\right)\right|<\arcsin \frac{\varkappa}{2}
$$

By using (56) and (58), we obtain

$$
\begin{aligned}
& \left|\arg \left(\frac{z d_{v, a, b, c}^{\prime}(z)}{d_{v, a, b, c}(z)}\right)\right| \\
& \quad=\left|\arg \left(\frac{z}{d_{v, a, b, c}(z)}\right)+\arg \left(d_{v, a, b, c}^{\prime}(z)\right)\right| \\
& \quad \leq\left|\arg \left(\frac{z}{d_{v, a, b, c}(z)}\right)\right|+\left|\arg \left(d_{v, a, b, c}^{\prime}(z)\right)\right| \\
& \quad<\arcsin \frac{\varkappa}{2}+\arcsin \varkappa \\
& \quad=\arcsin \left(\varkappa \sqrt{1-\frac{\varkappa^{2}}{4}}+\frac{\varkappa}{4} \sqrt{1-\varkappa^{2}}\right),
\end{aligned}
$$

which implies that $d_{v, a, b, c} \in \widetilde{\mathcal{S}^{*}}(\alpha)$ for $\alpha=$ (2/ $\pi) \arcsin \left(\varkappa \sqrt{1-\varkappa^{2} / 4}+(\varkappa / 4) \sqrt{1-\varkappa^{2}}\right)$.
Corollary 16. For $k=k^{*}$, where $k^{*}$ is the positive root of $2 k^{2}-$ $3 \sqrt{5} k-2 \sqrt{5}=0$, then $d_{v, a, b, c}(z) \in \mathcal{S}^{*}$.

\section{Close-to-Convexity with respect to Certain Functions}

Recently many authors discuss the close-to-convexity of some special functions with respect to certain functions. Here, we are also interested in the close-to-convexity of normalized Dini function.

Theorem 17. Let $a \in \mathbb{R}^{+}, b \in \mathbb{R}, c \in \mathbb{R}$, and $v \in \mathbb{R}$. If $\Gamma(v+$ $n+1) \geq-c(2 n+a) \Gamma(v+n) /(2(n-1)+a)$, then $d_{v, a, b, c}$ is close-to-convex with respect to $-\log (1-z)$.

Proof. Set

$$
f(z)=z+\sum_{n=2}^{\infty} b_{n-1} z^{n} .
$$

To prove that $f$ is close-to-convex with respect to the function $-\log (1-z)$, we use Lemma 5. Therefore, we have to prove that $\left\{n b_{n-1}\right\}_{n \geq 2}$ is a decreasing sequence. After some computations, we obtain

$$
\begin{aligned}
n b_{n-1}-(n+1) b_{n} & =\frac{n(-c)^{n-1}(2(n-1)+a) \Gamma(v+1)}{a 4^{n-1}(n-1) ! \Gamma(v+n)}-\frac{(n+1)(-c)^{n}(2 n+a) \Gamma(v+1)}{a 4^{n} n ! \Gamma(v+n+1)} \\
& =\frac{(-c)^{n-1} \Gamma(v+1)}{a 4^{n-1}(n-1) !}\left[\frac{4 n^{2} \Gamma(v+n+1)(2(n-1)+a)+c(n+1)(2 n+a) \Gamma(v+n)}{4 n \Gamma(v+n+1) \Gamma(v+n)}\right]>0
\end{aligned}
$$

By using the conditions on parameters, we easily observe that $n b_{n-1}-(n+1) b_{n}>0$ for all $n \geq 2$, and thus $\left\{n b_{n-1}\right\}_{n \geq 2}$ is a decreasing sequence. By Lemma 5 it follows that $f$ is close-to-convex with respect to the function $-\log (1-$ $z)$.

Theorem 18. Let $a \in \mathbb{R}^{+}, b \in \mathbb{R}, c \in \mathbb{R}$, and $v \in \mathbb{R}$. If $\Gamma(v+n+1) \geq-c(2 n+a) \Gamma(v+n) /(2(n-1)+a)$, then $d_{v, a, b, c}$ is close-to-convex with respect to $(1 / 2) \log ((1+z) /(1-$ $z)$ ).
Proof. Set

$$
f(z)=z+\sum_{n=2}^{\infty} B_{2 n-1} z^{2 n-1}
$$

Here $B_{2 n-1}=b_{n-1}=(-c)^{n-1}(2(n-1)+a) \Gamma(v+1) / a 4^{n-1}(n-$ $1) ! \Gamma(v+n+1)$, and therefore we have $b_{1}=-c(2+a) / 4 a k$ and $B_{2 n-1}>0$ for all $n \geq 2$. To prove our main result we will prove that $\left\{(2 n-1) b_{n-1}\right\}_{n \geq 2}$ is a decreasing sequence. After some computations, we obtain

$$
\begin{aligned}
(2 n-1) b_{n-1}-(2 n+1) b_{n} & =\frac{(2 n-1)(-c)^{n-1}(2(n-1)+a) \Gamma(v+1)}{a 4^{n-1}(n-1) ! \Gamma(v+n)}-\frac{(2 n+1)(-c)^{n}(2 n+a) \Gamma(v+1)}{a 4^{n} n ! \Gamma(v+n+1)} \\
& =\frac{(-c)^{n-1} \Gamma(v+1)}{a 4^{n-1}(n-1) !}\left[\frac{4 n(2 n-1) \Gamma(v+n+1)(2(n-1)+a)+c(2 n+1)(2 n+a) \Gamma(v+n)}{4 n \Gamma(v+n+1) \Gamma(v+n)}\right] \\
& >0 .
\end{aligned}
$$

By using the conditions on parameters we easily observe that $(2 n-1) b_{n-1}-(2 n+1) b_{n}>0$ for all $n \geq 2$, and thus $\left\{(2 n-1) b_{n-1}\right\}_{n \geq 2}$ is a decreasing sequence. By Lemma 5 it follows that $f(z)$ is close-to-convex with respect to the function $(1 / 2) \log ((1+z) /(1-z))$.

\section{Hardy Spaces of Dini Function}

Let $\mathscr{H}$ denote the class of all analytic functions in the open unit disk $\mathscr{U}=\{z:|z|<1\}$ and $\mathscr{H}^{\infty}$ denote the space of all bounded functions on $\mathscr{H}$. This is a Banach algebra with respect to the norm 


$$
\|f\|_{\infty}=\sup _{z \in \mathscr{U}}|f(z)| .
$$

We denote $\mathscr{H}^{p}, 0<p<\infty$ for the space of all functions $f \in \mathscr{H}$ such that $|f|^{p}$ admits a harmonic majorant. $\mathscr{H}^{p}$ is a Banach space if the norm of $f$ is defined to be $p$-th root of the least harmonic majorant of $|f|^{p}$ for some fixed $z \in \mathcal{U}$. Another equivalent definition of norm is given as follows: Let $f \in \mathscr{H}$, and set

$$
\begin{aligned}
& M_{p}(r, f) \\
& \quad= \begin{cases}\left(\frac{1}{2 \pi} \int_{0}^{2 \pi}\left|f\left(r e^{i \theta}\right)\right|^{p} d \theta\right)^{1 / p} & 0<p<\infty, \\
\max \{|f(z)|:|z| \leq r\}, & p=\infty .\end{cases}
\end{aligned}
$$

Then the function $f \in \mathscr{H}^{p}$ if $M_{p}(r, f)$ is bounded for all $r \in$ $[0,1)$. It is clear that

$$
\mathscr{H}^{\infty} \subset \mathscr{H}^{q} \subset \mathscr{H}^{p}, \quad 0<q<p<\infty .
$$

For some details see [20]. It is also known [20] that, for $\operatorname{Re}\left(f^{\prime}(z)\right)>0$ in $\mathcal{U}$, then

$$
\begin{aligned}
& f^{\prime} \in \mathscr{H}^{q}, \quad q<1, \\
& f \in \mathscr{H}^{q /(1-q)}, \quad 0<q<1 .
\end{aligned}
$$

Hardy spaces of hypergeometric functions are recently studied by Ponnusamy [21]. Baricz [2] uses the idea of Ponnusamy and found the Hardy spaces of Bessel functions while Yağmur and Orhan [10] studied the same problem for generalized Struve functions. Similarly Yağmur [22] studied the problem for Lommel functions and Raza et al. [9] studied the same problem for Wright functions.

To prove our main results we need the following lemmas.

Lemma 19 (see [23]). $\mathscr{P}_{0}(\alpha) * \mathscr{P}_{0}(\beta) \subset \mathscr{P}_{0}(\gamma)$, where $\gamma=$ $1-2(1-\alpha)(1-\beta)$ with $\alpha, \beta<1$ and the value of $\gamma$ is best possible.

Lemma 20 (see [24]). For $\alpha, \beta<1$ and $\gamma=1-2(1-\alpha)(1-\beta)$, we have $\mathscr{R}_{0}(\alpha) * \mathscr{R}_{0}(\beta) \subset \mathscr{R}_{0}(\gamma)$ or equivalently $\mathscr{P}_{0}(\alpha) *$ $\mathscr{P}_{0}(\beta) \subset \mathscr{P}_{0}(\gamma)$.

Lemma 21 (see [25]). If the function $f$, convex of order $\alpha$, where $\alpha \in[0,1)$, is not of the form

$$
f(z)= \begin{cases}\zeta+\eta\left(1-z e^{i \gamma}\right)^{2 \alpha-1}, & \alpha \neq \frac{1}{2}, \\ \zeta+\eta \log \left(1-z e^{i \gamma}\right), & \alpha \neq \frac{1}{2},\end{cases}
$$

for some complex numbers $\zeta$ and $\eta$ and for some real number $\gamma$ then the following statements hold:

(i) There exists $\delta=\delta(f)>0$, such that $f^{\prime} \in \mathscr{H}^{\delta+1 / 2(1-\alpha)}$.

(ii) If $\alpha \in[1,1 / 2)$, then there exists $\tau=\tau(f)>0$, such that $f \in \mathscr{H}^{\tau+1 /(1-2 \alpha)}$.

(iii) If $\alpha \geq 1 / 2$, then $f \in \mathscr{H}^{\infty}$.

The lemma defined below also plays an important role to find our main results.
Theorem 22. Let $\alpha \in[0,1)$ and $((4(k+1)-|c|)(4|c|+2 a|c|)+$ $\left.9|c|^{2}+2|c|^{2}(2+a)+a|c|^{2}\right) /((4(k+1)-|c|)(4 a k-4|c|-2 a|c|)-$ $\left.4|c|^{2}-2|c|^{2}(2+a)-a|c|^{2}\right)<1-\alpha$. Then

(i) $d_{v, a, b, c} \in \mathscr{H}^{1 / 1-2 \alpha}$ for $\alpha \in[0,1 / 2)$;

(ii) $d_{v, a, b, c} \in \mathscr{H}^{\infty}$ for $\alpha \geq 1 / 2$.

Proof. By using the definition of Hypergeometric function

$$
{ }_{2} F_{1}(a, b, c ; z)=\sum_{n=0}^{\infty} \frac{(a)_{n}(b)_{n}}{(c)_{n}} \frac{z^{n}}{n !},
$$

we have

$$
\begin{aligned}
\zeta+\frac{\eta z}{\left(1-z e^{i \gamma}\right)^{1-\alpha}} & =\zeta+\eta z_{2} F_{1}\left(1,1-2 \alpha, 1 ; z e^{i \gamma}\right) \\
& =\zeta+\eta \sum_{n=0}^{\infty} \frac{(1-2 \alpha)_{n}}{n !} e^{i \gamma n} z^{n+1}
\end{aligned}
$$

for $\zeta, \eta \in \mathbb{C}$, and $\alpha \neq 1 / 2$ and for $\gamma \in \mathbb{R}$. On the other hand

$$
\begin{aligned}
\zeta+\eta \log \left(1-z e^{i \gamma}\right) & =\zeta-\eta z_{2} F_{1}\left(1,1,2 ; z e^{i \gamma}\right) \\
& =\zeta-\eta \sum_{n=0}^{\infty} \frac{1}{n+1} e^{i \gamma n} z^{n+1}
\end{aligned}
$$

This implies that $d_{v, a, b, c}$ is not of the form $\zeta+\eta z\left(1-z e^{i \gamma}\right)^{2 \alpha-1}$ for $\alpha \neq 1 / 2$ and $\zeta+\eta \log \left(1-z e^{i \gamma}\right)$ for $\alpha=1 / 2$, respectively. Also, from part (ii) of Theorem $6, d_{v, a, b, c}$ is convex of order $\alpha$. Hence, by using Lemma 21, we have required result.

Theorem 23. Let $v>-0.8660254038$ and $f \in \mathscr{R}$. Then the convolution $d_{v} * f$ is in $\mathscr{H}^{\infty} \cap \mathscr{R}$.

Proof. Let $h(z)=d_{v} * f(z)$. Then it is clear that $h^{\prime}(z)=d_{v} / z *$ $f^{\prime}(z)$. Using Corollary 9 of part (iv), we have $d_{v} / z \in \mathscr{P}$. Since $f \in \mathscr{R}$, therefore by using Lemma $19 h \in \mathscr{R}$. It is also clear that $d_{v} / z$ is an entire function and therefor $h$ is entire. This implies that $h$ is bounded. Hence, we have the required result.

Theorem 24. Let $v \in \mathbb{R}$ with $(2|c|(k+1)+a|c|(k+1)) / a k(4(k+$ $1)-|c|)<1-\alpha$, and then $d_{v, a, b, c} / z \in \mathscr{P}(\alpha), \alpha \in[0,1)$, and $z \in \mathscr{U}$. If $f \in \mathscr{R}(\beta)$, with $\beta<1$, then $d_{v, a, b, c} * f \in \mathscr{R}(\gamma)$, where $\gamma=1-2(1-\alpha)(1-\beta)$.

Proof. Let $h(z)=d_{v, a, b, c}(z) * f(z)$, and then $h^{\prime}(z)=$ $d_{v, a, b, c}(z) / z * f^{\prime}(z)$. Now, from Theorem 6 of part (iv), we have $d_{v, a, b, c} / z \in \mathscr{P}(\alpha)$. By using Lemma 19 and the fact that $f^{\prime} \in \mathscr{P}(\beta)$, we have $h^{\prime}(z) \in \mathscr{P}(\gamma)$, where $\gamma=1-2(1-\alpha)(1-\beta)$. Consequently, we have $h \in \mathscr{R}(\gamma)$.

Corollary 25. Let $0 \leq \alpha<1,(2|c|(k+1)+a|c|(k+1)) / a k(4(k+$ $1)-|c|)<1-\alpha$, and then $d_{v, a, b, c} / z \in \mathscr{P}(\alpha)$. If $f \in \mathscr{R}(\beta)$, $\beta=(1-2 \alpha)(2-2 \alpha)$, then $d_{v, a, b, c} * f \in \mathscr{R}(0)$.

Corollary 26. Let $v>-0.8660254038$. If $f \in \mathscr{R}(1 / 2)$, then $d_{v} * f \in \mathscr{R}(0)$. 
By using Theorem 23 and Corollary 25, then we have the following corollary.

Corollary 27. If $f \in \mathscr{R}$, then the convolutions $d_{1 / 2} * f$ and $d_{3 / 2} * f$ are in $\mathscr{H}^{\infty} \cap \mathscr{R}$. Moreover, if $f \in \mathscr{R}(1 / 2)$, then $d_{1 / 2} * f$ and $d_{3 / 2} * f \in \mathscr{R}(0)$.

\section{Data Availability}

No data were used to support this study.

\section{Conflicts of Interest}

The authors declare that there are no conflicts of interest regarding the publication of this paper.

\section{Acknowledgments}

The work here is supported by UKM Grant no. GUP-2017064.

\section{References}

[1] I. Aktas, Á. Baricz, and N. Yagmur, "Bounds for the radii of univalence of some special functions," Mathematical Inequalities \& Applications, vol. 20, no. 3, pp. 825-843, 2017.

[2] Á. Baricz, "Bessel transforms and Hardy space of generalized Bessel functions," Mathematica, vol. 48, no. 71, pp. 127-136, 2006.

[3] Á. Baricz, Generalized Bessel Functions of the First Kind, vol. 1994 of Lecture Notes in Mathematics, Springer, Berlin, Germany, 2010.

[4] Á. Baricz, M. Çaglar, and E. Deniz, "Starlikeness of Bessel functions and their derivatives," Mathematical Inequalities \& Applications, vol. 19, no. 2, pp. 439-449, 2016.

[5] Á. Baricz, S. Ponnusamy, and S. Singh, "Modified Dini functions: monotonicity patterns and functional inequalities," Acta Mathematica Hungarica, vol. 149, no. 1, pp. 120-142, 2016.

[6] Á. Baricz and R. Szász, "The radius of convexity of normalized Bessel functions," Analysis Mathematica, vol. 41, no. 3, pp. 141151, 2015.

[7] M. U. Din, M. Raza, N. Yagmur, and S. N. Malik, "On partial sums of Wright functions," UPB Scientific Bulletin, Series A, vol. 80, no. 2, pp. 79-90, 2018.

[8] S. Kanas, S. R. Mondal, and A. D. Mohammed, "Relations between the generalized Bessel functions and the Janowski class," Mathematical Inequalities \& Applications, vol. 21, no. 1, pp. 165-178, 2018.

[9] M. Raza, M. U. Din, and S. N. Malik, "Certain Geometric Properties of Normalized Wright Functions," Journal of Function Spaces, vol. 2016, Article ID 1896154, 8 pages, 2016.

[10] N. Yagmur and H. Orhan, "Hardy space of generalized Struve functions," Complex Variables and Elliptic Equations, vol. 59, no. 7, pp. 929-936, 2014.

[11] G. N. Watson, A Treatise on the Theory of Bessel Functions, Cambridge University Press, Cambridge, UK, 2nd edition, 1944.

[12] E. Deniz, H. Orhan, and H. M. Srivastava, "Some sufficient conditions for univalence of certain families of integral operators involving generalized Bessel functions," Taiwanese Journal of Mathematics, vol. 15, no. 2, pp. 883-917, 2011.
[13] Á. Baricz, E. Deniz, and N. Yagmur, "Close-to-convexity of normalized Dini functions," Mathematische Nachrichten, vol. 289, no. 14-15, pp. 1721-1726, 2016.

[14] E. Deniz, Ş. Gören, and M. Çağlar, "Starlikeness and convexity of the generalized Dini functions," in AIP Conference Proceedings 1833, 020004, 2017.

[15] P. T. Mocanu, "Some starlike conditions for analytic functions," Revue Roumaine des Mathematiques Pures et Appliquees, vol. 33, pp. 117-124, 1988.

[16] V. Pescar, "A new generalization of Ahlfors's and Becker's criterion of univalence," Malaysian Mathematical Society (Second Series), vol. 19, no. 2, pp. 53-54, 1996.

[17] D. J. Hallenbeck and S. Ruscheweyh, "Subordination by convex functions," Proceedings of the American Mathematical Society, vol. 52, pp. 191-195, 1975.

[18] S. Owa, M. Nunokawa, H. Saitoh, and H. M. Srivastava, "Closeto-convexity, starlikeness, and convexity of certain analytic functions," Applied Mathematics Letters, vol. 15, no. 1, pp. 63-69, 2002.

[19] S. Ozaki, "On the theory of multivalent functions," Science Reports of the Tokyo Bunrika Daigaku, vol. 2, pp. 167-188, 1935.

[20] P. L. Duren, Theory of Hp Spaces, A series of Monographs and Textbooks in Pure and Applied Mathematics, vol. 38, Academic Press New York, London, UK, 1970.

[21] S. Ponnusamy, "The Hardy spaces of hypergeometric functions," Complex Variables, Theory and Application, vol. 29, no. 1, pp. 8396, 1996.

[22] N. Yagmur, "Hardy space of Lommel functions," Bulletin of the Korean Mathematical Society, vol. 52, no. 3, pp. 1035-1046, 2015.

[23] J. Stankiewicz and Z. Stankiewicz, "Some applications of the Hadamard convolution in the theory of functions," Annales Universitatis Mariae Curie-Sklodowska, vol. 40, pp. 251-265, 1987.

[24] S. Ponnusamy, "Inclusion theorems for convolution product of second order polylogarithms and functions with the derivative in a halfplane," Rocky Mountain Journal of Mathematics, vol. 28, no. 2, pp. 695-733, 1998.

[25] P. J. Eenigenburg and F. R. Keogh, "The Hardy class of some univalent functions and their derivatives," Michigan Mathematical Journal, vol. 17, pp. 335-346, 1970. 


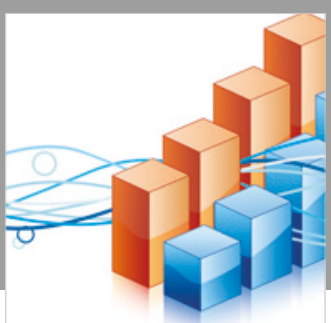

Advances in

Operations Research

\section{-n-m}
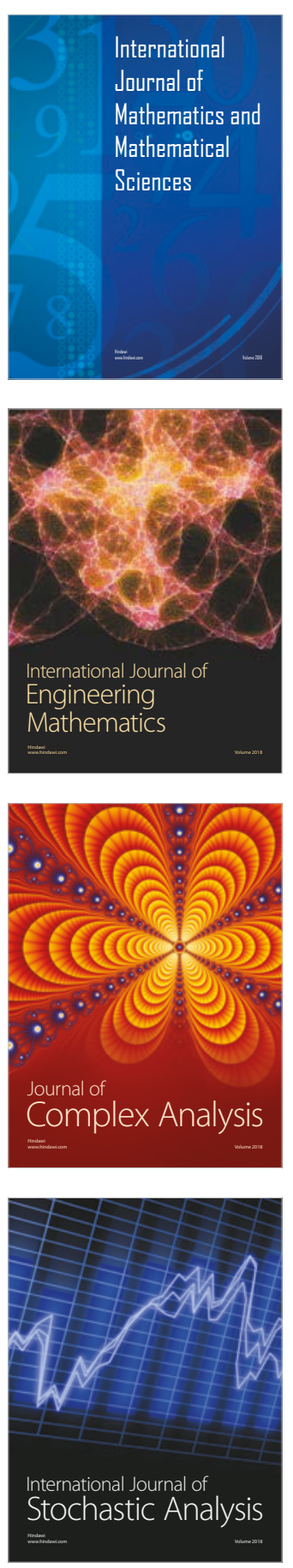
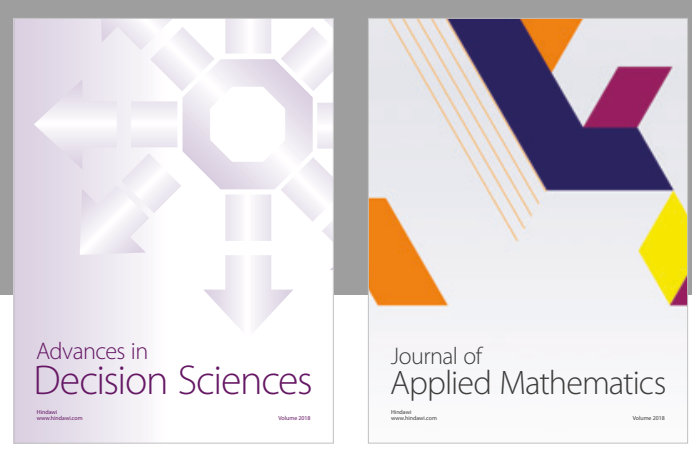

Journal of

Applied Mathematics
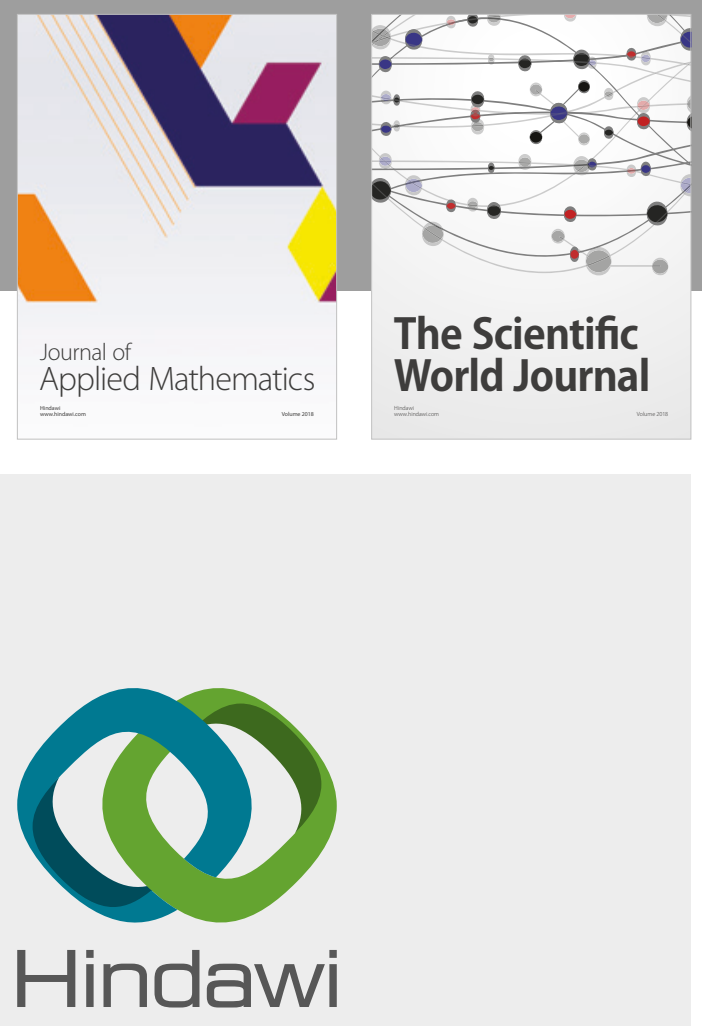

Submit your manuscripts at

www.hindawi.com

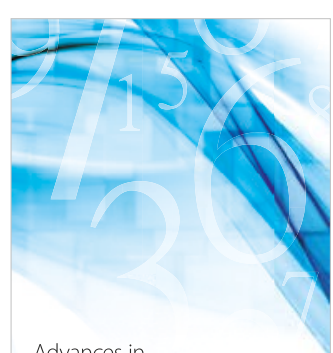

Advances in
Numerical Analysis
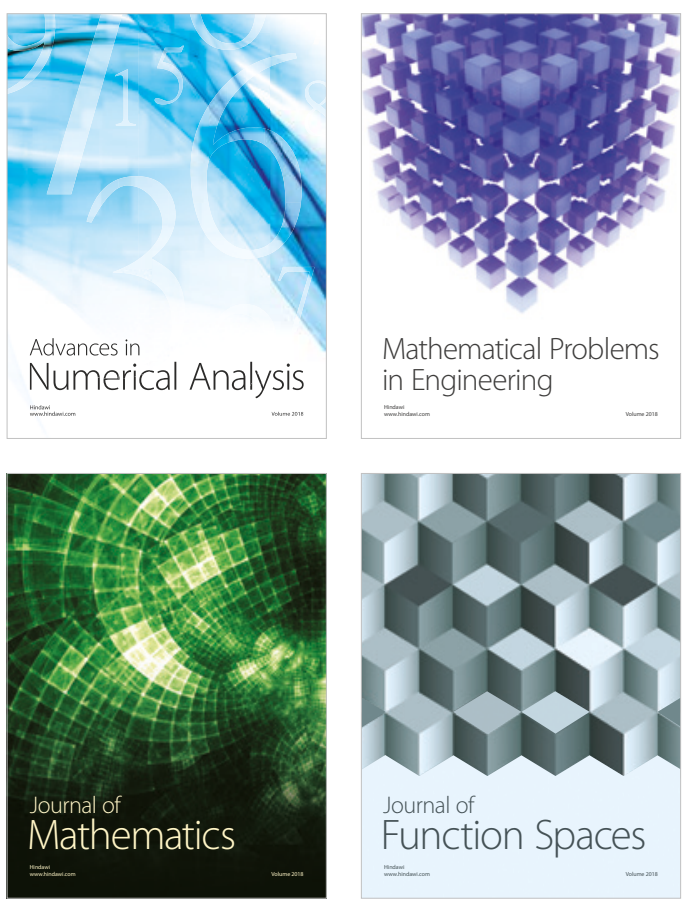

Mathematical Problems in Engineering

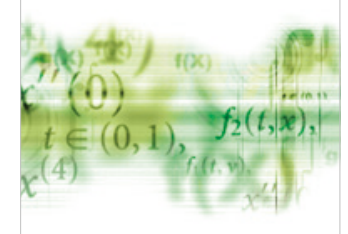

International Journal of

Differential Equations

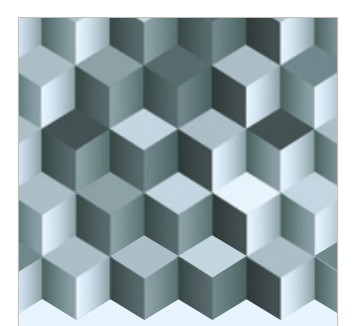

Journal of

Function Spaces

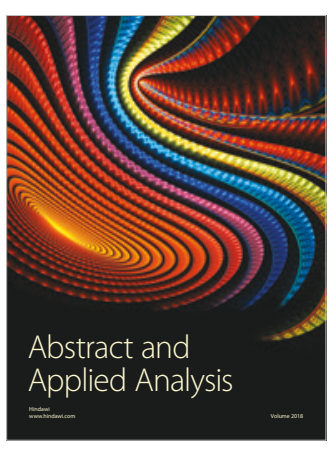

The Scientific

World Journal

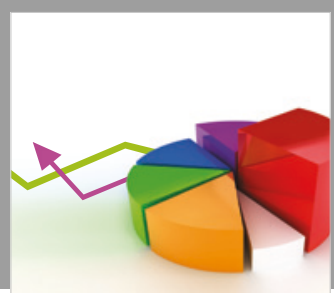

Journal of

Probability and Statistics
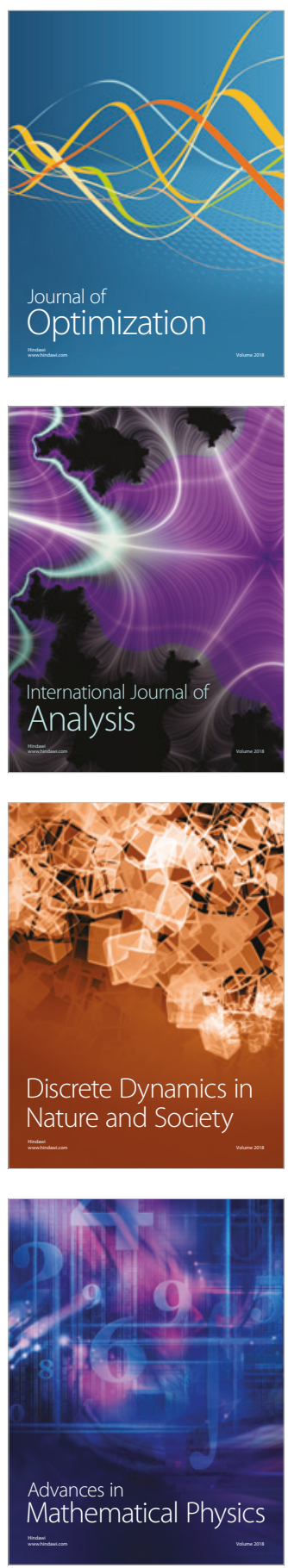